Article

\title{
Symmetries of Spatial Graphs and Rational Twists along Spheres and Tori
}

\section{Toru Ikeda}

Department of Mathematics, Faculty of Science, Kochi University, 2-5-1 Akebono-cho, Kochi-Shi, Kochi 780-8520, Japan; E-Mail: ikedat@ kochi-u.ac.jp

Received: 14 November 2011; in revised form: 12 January 2012 / Accepted: 13 January 2012 /

Published: 20 January 2012

\begin{abstract}
A symmetry group of a spatial graph $\Gamma$ in $S^{3}$ is a finite group consisting of orientation-preserving self-diffeomorphisms of $S^{3}$ which leave $\Gamma$ setwise invariant. In this paper, we show that in many cases symmetry groups of $\Gamma$ which agree on a regular neighborhood of $\Gamma$ are equivalent up to conjugate by rational twists along incompressible spheres and tori in the exterior of $\Gamma$.
\end{abstract}

Keywords: 3-manifold; geometric topology; symmetry; finite group action; spatial graph; rational twist

\section{Introduction}

There are several approaches to the theory of graphs embedded in the 3-sphere, which are often motivated by molecular chemistry, since the chemical properties of a molecule depend on the symmetries of its molecular bond graph (see, for example, [1]). The symmetries of an abstract graph $\Gamma$ are described by automorphisms. If $\Gamma$ is embedded in $S^{3}$, some of these automorphisms are induced from self-diffeomorphisms of $S^{3}$. For example, [2-6] studied the extendabilities of the automorphisms of $\Gamma$, mainly in the case of Möbius ladders, complete graphs, and 3-connected graphs.

Even if the automorphisms of $\Gamma$ extend to self-diffeomorphisms of $S^{3}$, we face the problem of the uniqueness of the extensions. In this situation, it is enough to consider $\Gamma$ to be a topological space, since we need to study self-diffeomorphisms of $S^{3}$ which agree on $\Gamma$. In the case of a non-torus knot in $S^{3}$, there are only finitely many conjugacy classes of symmetries (see [7,8]). For a cyclic period or a free period of a knot in $S^{3}$, it is shown in $[9,10]$ that the cyclic group generated by the periodic self-diffeomorphism of $S^{3}$ defining the symmetry is unique up to conjugate in some cases. Moreover, 
the author [11] generalized this result to the case of links in $S^{3}$. In this paper, we generalize these results to the case of symmetries of spatial graphs in $S^{3}$.

Suppose that any component of $\Gamma$ is a non-trivial graph with no leaf. We see $\Gamma$ as a geometric simplicial complex, and denote by $|\Gamma|$ the underlying topological space of $\Gamma$. A tame embedding of $|\Gamma|$ into $S^{3}$ is called a spatial embedding of $\Gamma$ into $S^{3}$, or simply a spatial graph $\Gamma$ in $S^{3}$. We say that $\Gamma$ is splittable if there exists a sphere in $S^{3}$ disjoint from $\Gamma$ that separates the components of $\Gamma$. We say that $\Gamma$ is non-splittable if it is not splittable. Suppose that an incompressible torus in $S^{3}-\Gamma$ bounds a solid torus $V$ in $S^{3}$ containing $\Gamma$. The core of $V$ is called a companion knot of $\Gamma$ if it is not ambient isotopic to $\Gamma$ in $V$. If there is no companion knot of $\Gamma$, every incompressible torus in $S^{3}-\Gamma$ separates the components of $\Gamma$.

Let $M$ be a 3-manifold, and $X$ a submanifold of $M$. Denote by $N(X)$ a regular neighborhood of $X$, and by $E(X)=M-\operatorname{int} N(X)$ the exterior of $X$. We refer to a finite subgroup $G$ of the diffeomorphism group $\operatorname{Diff}(M)$ as a finite group action on $M$. Finite group actions $G_{1}$ and $G_{2}$ on $M$ are equivalent (relative to $X$ ) if some $h \in \operatorname{Diff}(M)$ conjugates $G_{1}$ to $G_{2}$ (and restricts to the identity map on $X$ ). A symmetry group $G$ of a spatial graph $\Gamma$ in $S^{3}$ is a finite group action on the pair $\left(S^{3}, \Gamma\right)$ which preserves the orientation of $S^{3}$.

Let $S^{2}$ be the unit sphere in $\mathbb{R}^{3}$, and $S^{1}$ the unit circle in the $x y$-plane in $\mathbb{R}^{3}$. Denote by $\operatorname{Rot}_{\theta} \in \operatorname{Diff}\left(\mathbb{R}^{3}\right)$ the rotation about the $z$-axis through angle $\theta$. Suppose that $\sigma_{n} \in \operatorname{Diff}\left(S^{2} \times I\right)$ and $\tau_{n} \in \operatorname{Diff}\left(S^{1} \times S^{1} \times I\right)$, where $n \in \mathbb{R}$, is given by $\sigma_{n}(x, t)=\left(\operatorname{Rot}_{2 \pi n t}(x), t\right)$ and $\tau_{n}(x, y, t)=\left(\operatorname{Rot}_{2 \pi n t}(x), y, t\right)$. Let $F$ be a 2-sided sphere or torus embedded in a 3-manifold $M$. Split $M$ open along $F$ into a (possibly disconnected) 3-manifold $M_{F}$. Denote by $F_{-}$and $F_{+}$the boundary components of $M_{F}$ originated from $F$. An $n$-twist along $F$ is a discontinuous map on $M$ induced from a diffeomorphism on $M_{F}-F_{-}$which restricts to the identity map on $E\left(F_{+}\right)$and the map on $N\left(F_{+}\right)$conjugate to $\sigma_{n}$ or $\tau_{n}$ according as $F$ is a sphere or not. We say that the $n$-twist is rational if $n \in \mathbb{Q}$. Figure 1 illustrates a rotational symmetry of $S^{3}$ with a setwise invariant sphere $S$, and its conjugate by a $1 / 2$-twist along $S$.

Figure 1. Conjugation by a $1 / 2$-twist along a sphere $S$.
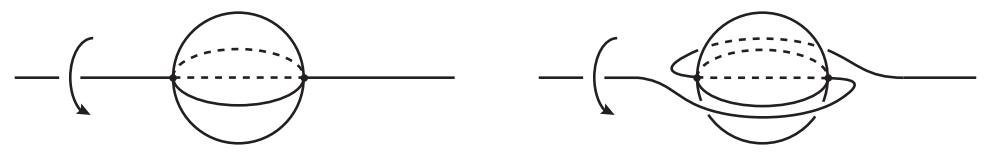

Our main theorem is the following:

Theorem 1.1. Let $\Gamma$ be a spatial graph in $S^{3}$ with no companion knot. Suppose that $G_{1}$ and $G_{2}$ are symmetry groups of $\Gamma$ such that

(1) $G_{1}(\gamma)=G_{2}(\gamma)=\gamma$ for at least one component $\gamma$ of $\Gamma$,

(2) either $\Gamma$ is non-splittable, or $G_{1}$ and $G_{2}$ are cyclic groups acting on $\Gamma$ freely, and

(3) $G_{1}$ and $G_{2}$ agree on $N(\Gamma)$.

Then there is a finite sequence of rational twists along incompressible spheres and tori in $E(\Gamma)$ whose composition conjugates $G_{2}$ to a symmetry group of $\Gamma$ equivalent to $G_{1}$ relative to $N(\Gamma)$. 
This paper is arranged as follows. In Section 2, we study symmetry groups of non-splittable spatial graph in terms of the equivariant JSJ decomposition of the exteriors. In Section 3, we establish a canonical version of the equivariant sphere theorem for the exteriors of spatial graphs with cyclic symmetry groups, and prove Theorem 1.1.

\section{Non-splittable Case}

For a non-splittable spatial graph $\Gamma$ in $S^{3}$ with a non-trivial symmetry group, there is a canonical method for splitting $E(\Gamma)$ equivariantly into geometric pieces by the loop theorem, the Dehn's lemma, and the JSJ decomposition theorem (see [12-14]).

Let $M$ be a Haken 3-manifold with incompressible boundary. The JSJ decomposition theorem and Thurston's uniformization theorem [15] assert that there is a canonical way of splitting the pair $(M, \partial M)$ along a disjoint, non-parallel, essential annuli and tori into pieces $\left(M_{i}, F_{i}\right)$ each of which is one of the following four types:

(1) $M_{i}$ is an $I$-bundle over a compact surface and $F_{i}$ is the $\partial I$-subbundle,

(2) $M_{i}$ admits a Seifert fibration in which $F_{i}$ is fibered,

(3) $\operatorname{int} M_{i}$ admits a complete hyperbolic structure of finite volume, and

(4) the double of $\left(M_{i}, F_{i}-\operatorname{int} \Phi_{i}\right)$ along a non-empty compact submanifold $\Phi_{i}$ of $F_{i}$ is of type (3).

For a finite group action $G$ on $M$, the fixed point set $\operatorname{Fix}(G)$ of $G$ is the set of points in $M$ each of which has the stabilizer $G$. The singular set $\operatorname{Sing}(G)$ of $G$ is the set of points in $M$ each of which has a non-trivial stabilizer.

Lemma 2.1. Let $T$ be a torus embedded in $S^{3}$. Suppose that $G_{1}$ and $G_{2}$ are orientation-preserving finite group actions on $S^{3}$ such that

(1) $G_{1}(N(T))=G_{2}(N(T))=N(T)$,

(2) $G_{1}$ and $G_{2}$ do not interchange the components of $\partial N(T)$, and

(3) $G_{1}$ and $G_{2}$ agree on $\partial N(T)$.

Then a rational twist along a component of $\partial N(T)$ conjugates $G_{2}$ to a finite group action $\widehat{G}_{2}$ on $S^{3}$ such that the actions of $G_{1}$ and $\widehat{G}_{2}$ on $N(T)$ are equivalent relative to $\partial N(T)$.

Proof. It is enough by Lemma 2.4 of [11] to consider the case where the actions of $G_{1}$ and $G_{2}$ on $N(T)$ are not free. For each $G_{i}$, Theorem 2.1 of [16] implies that $N(T) \cong T \times I$ admits a $G_{i}$-invariant product structure $\mathscr{P}_{i}$, in which $\operatorname{Sing}\left(G_{i}\right) \cap N(T)$ consists of $I$-fibers. Since each element of $G_{i}$ takes a meridian of $T$ to a meridian of $T$, the setwise stabilizer of each $I$-fiber is a trivial group or a 2-fold cyclic group. Therefore, the quotient space $N(T) / G_{i}$ admits the induced $I$-bundle structure over a 2-orbifold $B$ with underlying surface $F$ and $n$ cone points of index two. Since $T$ is a torus, the orbifold Euler characteristic $\chi_{\text {orb }}(B)$ of $B$ is calculated as follows (see [17]):

$$
\chi_{\mathrm{orb}}(B)=\chi(F)-n / 2=0 .
$$


Since $n>0, F$ is a sphere and $n=4$ holds.

Denote by $p_{i}: N(T) \rightarrow N(T) / G_{i}$ the projection map onto the quotient space for each $i$, and by $T_{t}$ the $T$-fiber $T \times\{t\}$ in $\mathscr{P}_{1}$. Connect the four cone points on $p_{1}\left(T_{0}\right)$ cyclically by a collection of arcs $\bar{a}_{1}, \bar{a}_{2}, \bar{a}_{3}$, and $\bar{a}_{4}$ with disjoint interiors. Each $\bar{a}_{i}$ lifts to an essential loop $a_{i}$ on $T_{0}$ such that $a_{i}$ and $a_{j}$ with $i \neq j$ are disjoint if $|j-i|=2$, and otherwise $a_{i}$ meets $a_{j}$ transversally in a point. Suppose that each $a_{i}$ is isotopic to a loop $b_{i}$ on $T_{1}$ along an annulus $B_{i}$ saturated by $I$-fibers in $\mathscr{P}_{1}$, and to a loop $c_{i}$ on $T_{1}$ along an annulus $C_{i}$ saturated by $I$-fibers in $\mathscr{P}_{2}$. Then the endpoints of each $p_{2}\left(c_{i}\right)$ is connected by $p_{2}\left(b_{j}\right)$ with $|i-j|=0$ or 2 . Since the underlying surface of $p_{2}\left(T_{1}\right)$ is a sphere, $\bigcup_{i=1}^{4} p_{2}\left(c_{i}\right)$ is isotopic to $\bigcup_{i=1}^{4} p_{2}\left(b_{i}\right)$ relative to the cone points. Therefore, $G_{2}\left(\bigcup_{i=1}^{4} C_{i}\right)$ is moved by an $G_{2}$-equivariant isotopy relative to $T_{0}$ so as to agree with $G_{1}\left(\bigcup_{i=1}^{4} B_{i}\right)$ on $T_{1}$.

The $I$-bundle structures in $\mathscr{P}_{2}$ and $\mathscr{P}_{1}$ respectively induce orbifold isomorphisms $\varphi_{1}: p_{2}\left(T_{1}\right) \rightarrow$ $p_{2}\left(T_{0}\right)$ and $\varphi_{2}: p_{2}\left(T_{0}\right) \rightarrow p_{2}\left(T_{1}\right)$ such that $\bar{h}=\varphi_{2} \circ \varphi_{1}$ setwise preserves the loop $\bigcup_{i=1}^{4} p_{2}\left(b_{i}\right)$. The restriction of $\bar{h}$ on $\bigcup_{i=1}^{4} p_{2}\left(b_{i}\right)$ is isotopic relative to the cone points to the identity map or an involution. Since $\bigcup_{i=1}^{4} p_{2}\left(b_{i}\right)$ splits $p_{2}\left(T_{1}\right)$ into two disks with no cone point, $\mathscr{P}_{2}$ is deformed by a $G_{2}$-equivariant isotopy so that afterwards $\bar{h}$ is the identity map or an involution.

Take an $\bar{h}$-invariant $S^{1}$-bundle structure $\mathscr{S}_{1}$ on $p_{2}\left(T_{1}\right)-p_{2}\left(b_{1} \cup b_{3}\right)$ with respect to which $p_{2}\left(b_{2}\right)$ and $p_{2}\left(b_{4}\right)$ are cross sectional, and an $\bar{h}$-invariant $S^{1}$-bundle structure $\mathscr{S}_{2}$ on $p_{2}\left(T_{1}\right)-p_{2}\left(b_{2} \cup b_{4}\right)$ with respect to which every fiber in $\mathscr{S}_{1}$ splits into two cross sections. Then $\mathscr{S}_{1}$ and $\mathscr{S}_{2}$ induce a $G_{2}$-invariant product structure $S^{1} \times S^{1}$ on $T_{1}$. Let $h: T_{1} \rightarrow T_{1}$ be the lift of $\bar{h}$ which takes each $c_{i}$ to $b_{i}$. Then we have $h=\operatorname{Rot}_{2 \pi m} \times \operatorname{Rot}_{2 \pi n}$ for some rational numbers $m$ and $n$.

Assume $(m, n) \neq(0,0)$. Take a rational number $\gamma$ so that $\gamma m$ and $\gamma n$ are coprime integers. Then $\alpha \gamma m+\beta \gamma n=1$ holds for some integers $\alpha$ and $\beta$. Let $\rho: \mathbb{R}^{2} \rightarrow S^{1} \times S^{1}$ be the covering map given by $\rho(x, y)=\left(\operatorname{Rot}_{2 \pi x}(1,0), \operatorname{Rot}_{2 \pi y}(1,0)\right)$. Denote by $\varphi$ the linear transformation on $\mathbb{R}^{2}$ represented by $\left(\begin{array}{cc}\alpha & \beta \\ -\gamma m & \gamma n\end{array}\right)$. Then the map $\rho \circ \varphi \circ \rho^{-1} \in \operatorname{Diff}\left(S^{1} \times S^{1}\right)$ conjugates $h$ to $\operatorname{Rot}_{2 \pi / \gamma} \times \operatorname{id}_{S^{1}}$. Thus, $h$ extends to $1 / \gamma$-twist $\tau$ along $T_{1}$. Since $h$ conjugates the action of $G_{2}$ on $T_{1}$ to itself, $\tau$ conjugates $G_{2}$ to a finite subgroup of $\operatorname{Diff}\left(S^{3}\right)$. Therefore, it is enough to consider the case $(m, n)=(0,0)$.

It is obvious that $h=\operatorname{Rot}_{2 \pi k} \times \operatorname{Rot}_{2 \pi l}$ holds for any integers $k$ and $l$. By verifying that, for some choice of $k$ and $l$, the above argument applied to $\operatorname{Rot}_{2 \pi k} \times \operatorname{Rot}_{2 \pi l}$ makes $G_{2}\left(\bigcup_{i=1}^{4} C_{i}\right)$ isotopic to $G_{1}\left(\bigcup_{i=1}^{4} B_{i}\right)$ relative to $\partial N(T)$, we may assume that they agree.

By considering an isotopy of $N(T)$ relative to $\partial N(T)$ which takes $\mathscr{P}_{2}$ to $\mathscr{P}_{1}$ on $\operatorname{Sing}\left(G_{1}\right) \cap N(T)$, we may assume that $G_{1}$ and $G_{2}$ agree on $\operatorname{Sing}\left(G_{1}\right) \cap N(T)$. Note that $\operatorname{Sing}\left(G_{1}\right) \cap N(T)$ splits $G_{1}\left(\bigcup_{i=1}^{4} B_{i}\right)$ into disks, and that $G_{1}\left(\bigcup_{i=1}^{4} B_{i}\right)$ splits $N(T)$ into balls. Then the identity map on $p_{2}\left(\operatorname{Sing}\left(G_{2}\right) \cap N(T)\right)$ extends to an orbifold isomorphism $\psi: p_{2}\left(\bigcup_{i=1}^{4} C_{i}\right) \rightarrow p_{1}\left(\bigcup_{i=1}^{4} B_{i}\right)$. Since the quotient space of any finite group action on $D^{3}$ is isomorphic to one of the orbifolds listed on page 191 of [15], $\psi$ and the identity map on $p_{2}(\partial N(T))$ extend to an orbifold isomorphism $p_{2}(N(T)) \rightarrow p_{1}(N(T))$. Thus, $G_{1}$ and $G_{2}$ are equivalent relative to $\partial N(T)$. Hence, the conclusion follows.

Lemma 2.2. Let $M$ be a Seifert manifold in $S^{3}$ with non-empty boundary, and $F$ a non-empty closed submanifold of $\partial M$. Suppose that $G_{1}$ and $G_{2}$ are finite group actions on $S^{3}$ such that

(1) $G_{1}(M)=G_{2}(M)=M$ and $G_{1}(F)=G_{2}(F)=F$, 
(2) $G_{1}(T)=G_{2}(T)=T$ for at least one component $T$ of $F$,

(3) $G_{1}$ and $G_{2}$ induce the same permutation on the set of the components of $\partial M$, and

(4) $G_{1}$ and $G_{2}$ agree on $F$.

Then there is a finite sequence of rational twists along incompressible tori in $M$ whose composition conjugates $G_{2}$ to a finite group action $\widehat{G}_{2}$ on $S^{3}$ such that the actions of $G_{1}$ and $\widehat{G}_{2}$ on $M$ are equivalent relative to $F$.

Proof. The case $M=D^{2} \times S^{1}$ and $F=\partial M$, the case $M=S^{1} \times S^{1} \times I$ and $F=\partial M$, and the case $M=S^{1} \times S^{1} \times I$ and $F \neq \partial M$ respectively follow from Lemma 2.1 of [11], Lemma 2.1 of this paper, and Theorem 8.1 of [16]. We therefore exclude these cases.

Denote by $\bigcup_{k} \xi_{k}$ the system of the exceptional fibers $\xi_{k}$ in $M$. Let $N\left(\xi_{k}\right)$ be a fibered regular neighborhood of each $\xi_{k}$. It follows from Theorem 2.2 of [16] that each $G_{i}$ preserves some Seifert fibration $\mathscr{S}_{i}$ of $M$. Then the uniqueness of a Seifert fibration of $M$ (see VI.18.Theorem of [12]) implies that $\bigcup_{k} N\left(\xi_{k}\right)$ is isotopic to a setwise $G_{i}$-invariant fibered regular neighborhood of the system of exceptional fibers in $\mathscr{S}_{i}$. Since Lemma 3.1 of [11] implies that the orders of the exceptional fibers are pairwise coprime, we may assume that $G_{1}\left(N\left(\xi_{k}\right)\right)=G_{2}\left(N\left(\xi_{k}\right)\right)=N\left(\xi_{k}\right)$ for each $k$. Therefore, it is enough by Lemma 2.1 of [11] to consider the case where $M$ is a product $S^{1}$-bundle.

It follows from Theorem 2.1 of [16] that $M$ admits a $G_{1}$-invariant product structure $\mathscr{P}_{1}$. If $F=\partial M$, $M$ admits a $G_{2}$-invariant product structure $\mathscr{P}_{2}$ which agrees with $\mathscr{P}_{1}$ on $F$ (see Theorem 2.3 of [16]). If $F \neq \partial M$, we see $M$ as the quotient of the double $\bar{M}$ of $M$ along $\partial M-F$ by $\mathbb{Z}_{2}$ generated by an orientation-reversing involution, and apply the same argument to the finite group action on $\bar{M}$, which is the extension of $\mathbb{Z}_{2}$ by $G_{2}$. Then we obtain a $G_{2}$-invariant product structure $\mathscr{P}_{2}$ of $M$ which agrees with $\mathscr{P}_{1}$ on $F$.

By the uniqueness of the $S^{1}$-bundle structure of $M$ (see VI.18.Theorem of [12]), there is a map $\varphi \in \operatorname{Diff}(M)$ isotopic to the identity which takes the $S^{1}$-bundle structure induced by $\mathscr{P}_{1}$ to the $S^{1}$-bundle structure induced by $\mathscr{P}_{2}$. Modify $\varphi$ in $\mathscr{P}_{2}$ by a fiber preserving isotopy in a fibered regular neighborhood of $F$ so as to restrict to the identity map on $F$. By conjugating $G_{2}$ by $\varphi$, we may therefore assume that $\mathscr{P}_{1}$ and $\mathscr{P}_{2}$ induce the same $S^{1}$-bundle structure of $M$.

Let $p: M \rightarrow B$ be the projection map onto the base surface $B$. Each $G_{i}$ induces a finite group action $\bar{G}_{i}$ on $B$. We consider $B$ to be lying on $S^{2}$. Then each $\bar{G}_{i}$ extends to an action on $S^{2}$. Since $\bar{G}_{1}$ and $\bar{G}_{2}$ agree on $p(F)$, the quotient spaces $B / \bar{G}_{1}$ and $B / \bar{G}_{2}$ are orbifold isomorphic to suborbifolds of the same spherical orbifold listed on page 188 of [15]. We may assume that $\bar{G}_{1}$ and $\bar{G}_{2}$ are not orientation-preserving, otherwise the conclusion follows from Lemma 3.2 and Remark 3.3 of [11]. Then the assumption $G_{1}(T)=G_{2}(T)=T$ implies that each $\bar{G}_{i}$ is generated by the reflection of $S^{2}$ in a loop. Since $G_{1}$ and $G_{2}$ permute the components of $\partial M$ similarly, $\partial B$ consists of loops $\ell_{1}, \ldots, \ell_{2 k}, \ell_{1}^{\prime}, \ldots, \ell_{n}^{\prime}$ such that

(1) $\bar{G}_{1}$ and $\bar{G}_{2}$ interchange $\ell_{2 i-1}$ and $\ell_{2 i}$ for $1 \leq i \leq k$, and

(2) $\bar{G}_{1}$ and $\bar{G}_{2}$ setwise preserve $\ell_{i}^{\prime}$ for $1 \leq i \leq n$. 
Without loss of generality, $\ell_{1}^{\prime}=p(T)$. Denote by $\operatorname{Fix}\left(\bar{G}_{i}\right)$ the fixed point circle of the action of each $\bar{G}_{i}$ on $S^{2}$. Suppose that each $\operatorname{Fix}\left(\bar{G}_{i}\right)$ is equipped with an orientation, and splits $B$ into two pieces $B_{i, 1}$ and $B_{i, 2}$ so that $\ell_{1}^{\prime} \cap B_{1,1}=\ell_{1}^{\prime} \cap B_{2,1}$ and $\ell_{1}^{\prime} \cap B_{1,2}=\ell_{1}^{\prime} \cap B_{2,2}$. We may assume without loss of generality that $\ell_{2 i-1} \subset B_{1,1}$ and $\ell_{2 i} \subset B_{1,2}$ for $1 \leq i \leq k$, and that we meets $\ell_{1}^{\prime}, \ldots, \ell_{n}^{\prime}$ in order as we go along $\operatorname{Fix}\left(\bar{G}_{1}\right)$.

Suppose $\ell_{2 i-1} \subset B_{2,2}$ and $\ell_{2 i} \subset B_{2,1}$ for some $i$. By taking a proper arc on $B / \bar{G}_{2}$ connecting $\ell_{2 i} / \bar{G}_{2}$ and $\operatorname{Fix}\left(\bar{G}_{2}\right) / \bar{G}_{2}$, we obtain a setwise $\bar{G}_{2}$-invariant arc $\alpha$ on $B$ which meets $\operatorname{Fix}\left(\bar{G}_{2}\right)$ in a point and connects $\ell_{2 i-1}$ and $\ell_{2 i}$. Then $\operatorname{Fix}\left(\bar{G}_{2}\right)$ is modified by the half twist along the loop $\partial N\left(\ell_{2 i-1} \cup \ell_{2 i} \cup \alpha\right) \cap \operatorname{int} B$, denoted by $\lambda$, so that afterwards $\ell_{2 i-1} \subset B_{2,1}$ and $\ell_{2 i} \subset B_{2,2}$, as illustrated in Figure 2. The argument presented for the proof of Lemma 2.1 implies that this modification is realized by a $1 / 2$-twist along the torus $p^{-1}(\lambda)$ which conjugates $G_{2}$ to a subgroup of $\operatorname{Diff}\left(S^{3}\right)$. We may therefore assume $\ell_{2 i-1} \subset B_{2,1}$ and $\ell_{2 i} \subset B_{2,2}$ for $1 \leq i \leq k$.

Figure 2. Half twist along $\lambda$.
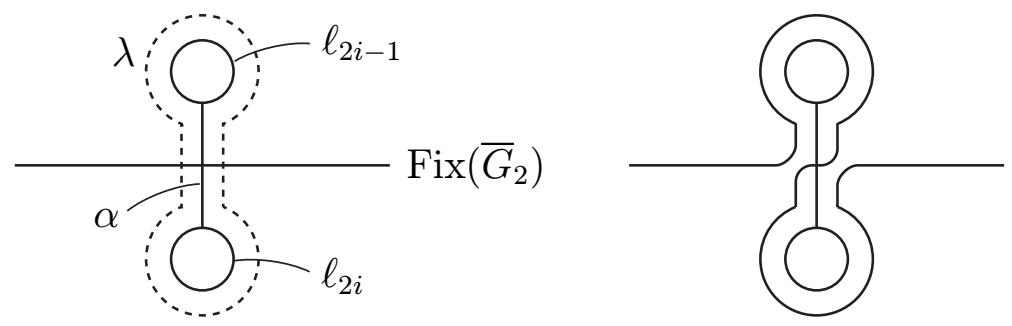

Suppose that $\ell_{i}^{\prime}$ and $\ell_{j}^{\prime}$ are connected by an arc $\alpha^{\prime}$ in $\operatorname{Fix}\left(\bar{G}_{2}\right) \cap B$. Then $\operatorname{Fix}\left(\bar{G}_{2}\right)$ is modified by the half twists along the loop $\lambda^{\prime}=\partial N\left(\ell_{i}^{\prime} \cup \ell_{j}^{\prime} \cup \alpha^{\prime}\right) \cap \operatorname{int} B$ so as to meet $\ell_{i}^{\prime}$ and $\ell_{j}^{\prime}$ in the reverse order, as illustrated in Figure 3, which is realized by the conjugation of $G_{2}$ by a $1 / 2$-twist along the torus $p^{-1}\left(\lambda^{\prime}\right)$, as before. Since every permutation on the set $\left\{\ell_{2}^{\prime}, \ldots, \ell_{n}^{\prime}\right\}$ is a product of transpositions, we may assume that $\operatorname{Fix}\left(\bar{G}_{2}\right)$ meets $\ell_{1}^{\prime}, \ldots, \ell_{n}^{\prime}$ in order. Moreover, we can change the order in which $\operatorname{Fix}\left(\bar{G}_{2}\right)$ meets the two points in $\ell_{i}^{\prime} \cap \operatorname{Fix}\left(\bar{G}_{2}\right)$ by the half twists along $\ell_{i}^{\prime}$, which is also realized by a $1 / 2$-twist along the torus $p^{-1}\left(\ell_{i}^{\prime}\right)$. We may therefore assume that $\bar{G}_{2}$ is equivalent to $\bar{G}_{1}$ relative to $\partial B$.

Figure 3. Half twist along $\lambda^{\prime}$.
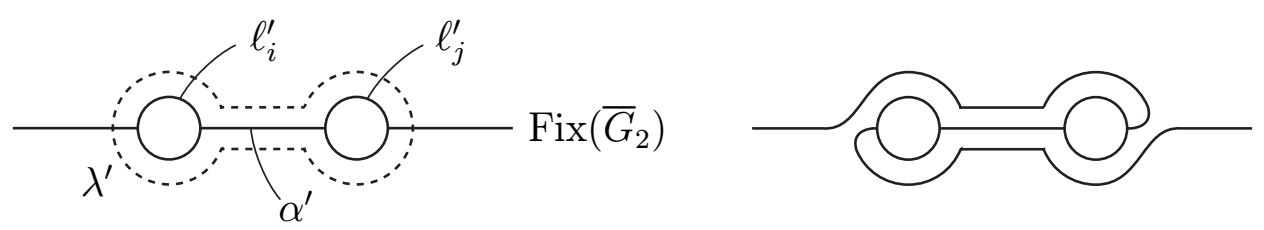

Now we may assume $\bar{G}_{1}=\bar{G}_{2}$. Take a map $h \in \operatorname{Diff}(M)$ which restricts to the identity map on $F$ and takes $\mathscr{P}_{2}$ to $\mathscr{P}_{1}$ setwise preserving every $S^{1}$-fiber. It is easy to verify that $h$ is extendable to a map in $\operatorname{Diff}\left(S^{3}\right)$. Hence, the conclusion follows by conjugating $G_{2}$ by $h$.

Lemma 2.3. Let $M$ be a compact connected 3-manifold in $S^{3}$ with non-empty boundary whose interior admits a complete hyperbolic structure of finite volume, and F a non-empty closed submanifold of $\partial M$. Suppose that $G_{1}$ and $G_{2}$ are finite group actions on $S^{3}$ such that 
(1) $G_{1}(M)=G_{2}(M)=M$ and $G_{1}(F)=G_{2}(F)=F$,

(2) $G_{1}$ and $G_{2}$ induce the same permutation on the set of the components of $\partial M$, and

(3) $G_{1}$ and $G_{2}$ agree on $F$.

Then there is a sequence of rational twists along tori in $F$ whose composition conjugates $G_{2}$ to a finite group action $\widehat{G}_{2}$ such that the actions of $G_{1}$ and $\widehat{G}_{2}$ on $M$ is equivalent relative to $F$.

Proof. It follows from Theorem 5.5 of [18] that int $M$ admits two complete hyperbolic structures of finite volume, one is $G_{1}$-invariant and the other is $G_{2}$-invariant. Mostow's rigidity theorem [15] implies that complete hyperbolic structures of finite volume on int $M$ are unique up to isometry representing the identity map on $\operatorname{Out}\left(\pi_{1}(M)\right)$. We may therefore assume that $\operatorname{int} M$ is endowed with the $G_{1}$-invariant hyperbolic structure, and that $G_{2}$ is conjugate to an isometric action $G_{2}^{\prime}$ by $h \in \operatorname{Diff}(M)$ which is isotopic to the identity map.

Next, we are going to modify $h$ in a regular neighborhood of $F$ so as to restrict to the identity map on $F$. It follows from Propostition D.3.18 of [19] that $F$ consists of tori. Let $h_{t}$ be an isotopy from $h$ to the identity map. Denote by $\bar{G}_{2}$ the finite group action on $F \times I$ whose restriction on $F \times\{t\}$ is induced from the finite group action on $F$ given by the conjugate of $G_{2}$ by $h_{t}$. In particular, the actions of $\bar{G}_{2}$ on $F \times\{0\}$ and $F \times\{1\}$ are respectively given by $G_{2}^{\prime}$ and $G_{2}$. Note that $\bar{G}_{2}$ preserves the product structure $F \times \partial I$, and that we can embed $F \times I$ in $S^{3}$ so that $\bar{G}_{2}$ extends to a finite group action on $S^{3}$.

We consider the partition of the set of the components of $F$ into the orbits under the permutation induced by $G_{2}$. Suppose that the orbits are represented by $T_{1}, \ldots, T_{n}$. Lemma 2.1 implies that a rational twist along $T_{i} \times\{1\}$ conjugates the setwise stabilizer of $T_{i} \times I$ in $\bar{G}_{2}$ so that the action on $T_{i} \times I$ is equivalent relative to $T_{i} \times \partial I$ to the action which preserves the product structure. Suppose that the rational twists along the tori in $F \times\{1\}$ are equivariantly induced from those along $T_{1} \times\{1\}, \ldots, T_{n} \times\{1\}$. By conjugating $\bar{G}_{2}$ by their composition, it is equivalent relative to $F \times \partial I$ to the action which preserves the product structure. This implies that $h$ is modified equivariantly so as to restrict to the identity map on $F$.

Suppose that $g_{1} \in G_{1}$ and $g_{2} \in G_{2}$ agree on $F$. Then $g_{1} \circ g_{2}^{-1}$ restricts to the identity map on $F$. Since the isometry group of int $M$ is finite (see [15]), Newman's theorem [20] implies $g_{1}=g_{2}$. Hence, $G_{1}$ and $G_{2}^{\prime}$ agree on $M$. This completes the proof.

Lemma 2.4. Let $M$ be a compact connected 3-manifold in $S^{3}$ with non-empty boundary such that the double $\bar{M}$ of $M$ along a non-empty compact submanifold $\Phi$ of $\partial M$ admits a complete hyperbolic structure of finite volume in its interior. Let $F$ be a closed submanifold of $\partial M$ containing $\Phi$. Suppose that $G_{1}$ and $G_{2}$ are finite group actions on $S^{3}$ such that

(1) $G_{1}(M)=G_{2}(M)=M$ and $G_{1}(F)=G_{2}(F)=F$,

(2) $G_{1}$ and $G_{2}$ induce the same permutation on the set of the components of $\partial M$, and

(3) $G_{1}$ and $G_{2}$ agree on $F$.

Then there is a finite sequence of rational twists along tori in $F$ whose composition conjugates $G_{2}$ to a finite group action $\widehat{G}_{2}$ such that the actions of $G_{1}$ and $\widehat{G}_{2}$ on $M$ are equivalent relative to $F$. 
Proof. We see $M$ as the quotient of $\bar{M}$ by $\mathbb{Z}_{2}$ generated by an orientation-reversing involution. Each $G_{i}$ induces a finite group action $\bar{G}_{i}$ on $\bar{M}$ which is an extension of $\mathbb{Z}_{2}$ by $G_{i}$. As in the proof of Lemma 2.3, we consider int $\bar{M}$ endowed with a $\bar{G}_{1}$-invariant hyperbolic structure. Then some $\bar{h} \in \operatorname{Diff}(\bar{M})$, which is isotopic to the identity map, conjugates $\bar{G}_{2}$ to an isometric action $\bar{G}_{2}^{\prime}$. Clearly, $\Phi$ meets $\operatorname{int} \bar{M}$ in a totally geodesic surface, and therefore $\bar{h}(\Phi)=\Phi$ holds.

Suppose that $\bar{g}_{1} \in \bar{G}_{1}$ and $\bar{g}_{2} \in \bar{G}_{2}^{\prime}$ respectively induce $g_{1} \in G_{1}$ and $g_{2} \in G_{2}$ which agree on $F$. Then $\bar{g}_{1}^{-1} \circ \bar{g}_{2}$ restricts to an isometry on each component $\Phi_{i}$ of $\Phi$, which is a compact surface of negative Euler characteristic (see Propostition D.3.18 of [19]). Since $\bar{g}_{1}^{-1} \circ \bar{g}_{2}$ is trivial in Out $\left(\pi_{1}\left(\Phi_{i}\right)\right), \bar{g}_{1}$ and $\bar{g}_{2}$ agree on $\Phi_{i}$. Therefore, [20] implies $g_{1}=g_{2}$. Hence, some $h \in \operatorname{Diff}(M)$, which setwise preserves $\Phi$ and is isotopic to the identity map, conjugates the action of $G_{1}$ on $M$ to $G_{2}$.

It follows from Proposition D.3.18 of [19] that $F-\Phi$ consists of tori. As in the proof of Lemma 2.3, modify $h$ in $N(F-\Phi)$ by rational twists along tori in $F-\Phi$ so that afterwards $h$ restricts to the identity map on $F-\Phi$ and conjugates the action of $G_{1}$ on $M$ to $G_{2}$. Moreover, we may assume by Lemma 2.3 of [11] that $h$ restricts to the identity map on $\Phi$. Since $h$ extends to an automorphism of $S^{3}$ which is diffeomorphic outside $M$, the conclusion follows.

Proposition 2.5. Theorem 1.1 is true, if $\Gamma$ is non-splittable.

Proof. The equivariant loop theorem (see Chapter VII of [15] and [21]) implies that there is a $G_{1}$-invariant system $\mathscr{D}_{1}$ of disjoint disks properly embedded in $E(\Gamma)$ which splits $E(\Gamma)$ into pieces with incompressible boundary. The equivariant Dehn's lemma [21,22] implies that the boundary loops of $\mathscr{D}_{1}$ bound a $G_{2}$-invariant system $\mathscr{D}_{2}$ of disjoint disks properly embedded in $E(\Gamma)$. Since $\Gamma$ is non-splittable, $E(\Gamma)$ is irreducible. Therefore, there is an isotopy of $E(\Gamma)$ relative to $\partial E(\Gamma)$ which takes $\mathscr{D}_{2}$ to $\mathscr{D}_{1}$. Since any finite group action on $D^{2}$ is orthogonal [15], we may assume that $G_{1}$ and $G_{2}$ agree on $\mathscr{D}_{1}$. Moreover, the induced actions on the balls obtained by splitting $E(\Gamma)$ along $\mathscr{D}_{1}$ are equivalent relative to the boundary (see [15]). Therefore, it is enough to consider the case where $E(\Gamma)$ is a Haken manifold with incompressible boundary.

We may assume by the equivariant JSJ decomposition theorem (see Theorem 8.6 of [16]) and by the uniqueness of the JSJ decomposition [13,14] that there is a $G_{1}$-invariant and $G_{2}$-invariant system $\mathscr{T}$ of essential annuli and tori in $E(\Gamma)$ realizing the canonical JSJ decomposition of the pair $(E(\Gamma), \partial E(\Gamma))$.

The argument presented for the proof of Proposition 3.10 of [11] implies that some $h \in \operatorname{Diff}\left(S^{3}\right)$, which is isotopic to the identity map relative to $N(\Gamma)$, conjugates $G_{2}$ to a finite group action which agree with $G_{1}$ on the annuli in $\mathscr{T}$. We may therefore assume that $\mathscr{T}$ contains no annuli.

The rest of the proof proceeds by induction on the number of tori in $\mathscr{T}$. Take a piece $M_{k}$ attaching $\partial E(\Gamma)$. By Lemmas 2.2, 2.3 and 2.4, it is enough to consider the case where $G_{2}$ agrees with $G_{1}$ on $G_{1}\left(M_{k}\right)$. Moreover, we may assume by Lemma 2.1 that $G_{1}$ and $G_{2}$ agree on the components of $\operatorname{cl}\left(E(\Gamma)-G_{1}\left(M_{k}\right)\right)$ each of which is a product $I$-bundle over a torus. Hence, the conclusion follows by the induction hypothesis.

\section{Possibly Splittable Case}

For a symmetry group $G$ of a splittable spatial graph $\Gamma$ in $S^{3}$, there is a setwise $G$-invariant system $\mathscr{S}$ of spheres realizing the prime factorization of $E(\Gamma)$ (see [23]). However, $\mathscr{S}$ is not unique in contrast 
to the JSJ decomposition of a Haken 3-manifold. If some component of $\Gamma$ is setwise invariant and every essential sphere in $E(\Gamma)$ has a trivial stabilizer, there is a canonical choice of $\mathscr{S}$ (see [11]). We first prove that this is possible also in the setting of Theorem 1.1.

Lemma 3.1. Let $\Gamma$ be a splittable spatial graph in $S^{3}$. Suppose that $G_{1}$ and $G_{2}$ are symmetry groups of $\Gamma$ such that

(1) $G_{1}(\gamma)=G_{2}(\gamma)=\gamma$ for at least one component $\gamma$ of $\Gamma$,

(2) $G_{1}$ and $G_{2}$ are cyclic groups acting on $\Gamma$ freely, and

(3) $G_{1}$ and $G_{2}$ agree on $N(\Gamma)$.

Then each $G_{i}$ admits a setwise $G_{i}$-invariant system $\mathscr{B}_{i}$ of disjoint balls in $S^{3}$ not containing $\gamma$ such that each $\partial \mathscr{B}_{i}$ realizes the prime factorization of $E(\Gamma)$. Moreover, for some choice of $\mathscr{B}_{1}$ and $\mathscr{B}_{2}$, there is a finite sequence of rational twists along incompressible tori in $E(\Gamma)-\operatorname{int} \mathscr{B}_{2}$ and a map in $\operatorname{Diff}\left(S^{3}\right)$ which restricts to the identity map on $N(\Gamma)$ whose composition conjugates the action of $G_{2}$ on $S^{3}-\operatorname{int} \mathscr{B}_{2}$ to the action of $G_{1}$ on $S^{3}-\operatorname{int} \mathscr{B}_{1}$.

Proof. Denote by $\Gamma_{\gamma}$ the non-splittable spatial subgraph of $\Gamma$ containing $\gamma$ which is obtained by the prime factorization of $E(\Gamma)$. It follows from the equivariant sphere theorem [23] that each $G_{i}$ admits a setwise $G_{i}$-invariant system $\mathscr{S}_{i}=S_{i, 1} \cup \cdots \cup S_{i, n}$ of disjoint, non-parallel, essential spheres in $E(\Gamma)$ realizing the prime factorization. Suppose that each $S_{i, j}$ bounds a ball $B_{i, j}$ disjoint from $\gamma$. Note that $\operatorname{Sing}\left(G_{i}\right)$ avoids $B_{i, j}$ or meets $B_{i, j}$ in a trivial 1-string tangle (see [15]).

Suppose $B_{i, j} \subset B_{i, k}$ for some distinct $j$ and $k$. Denote by $p_{i}: S^{3} \rightarrow S^{3} / G_{i}=S^{3}$ the projection map onto the quotient space. Take an arc $\alpha$ properly embedded in $p_{i}\left(B_{i, k}-\operatorname{int} B_{i, j}\right)$ which connects $p_{i}\left(\partial B_{i, k}\right)$ and $p_{i}\left(\partial B_{i, j}\right)$. Suppose that $\alpha$ lies on $p_{i}\left(\operatorname{Sing}\left(G_{i}\right)\right)$ if $\operatorname{Sing}\left(G_{i}\right)$ connects $\partial B_{i, j}$ and $\partial B_{i, k}$. By replacing $B_{i, j}$ with another ball in int $B_{i, k}$ if necessary, $\alpha$ meets $\mathscr{S}_{i}$ in its endpoints. By drilling into $p_{i}\left(B_{i, k}\right)$ along $\alpha \cup p_{i}\left(B_{i, j}\right), B_{i, k}$ is deformed to a ball disjoint from $B_{i, j}$, as illustrated in Figure 4 in which the result of the deformation is presented in a cross-sectional view. By a finite repetition of this operation, we obtain a system $\mathscr{B}_{i}=B_{i, 1} \cup \cdots \cup B_{i, n}$ of disjoint balls. This proves the first half of the lemma. Without loss of generality, $\Gamma \cap B_{2, j}=\Gamma \cap B_{1, j}$ for $1 \leq j \leq n$.

Figure 4. Modification of $p_{i}\left(B_{i, k}\right)$ which makes $p_{i}\left(B_{i, k}\right)$ disjoint from $p_{i}\left(B_{i, j}\right)$.
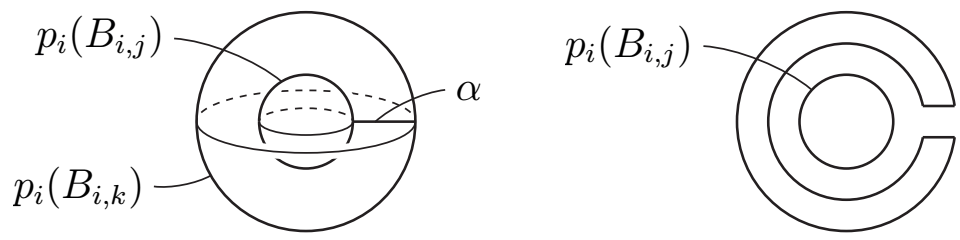

Proposition 2.5 implies that there is a finite sequence of rational twists along incompressible tori in $E\left(\Gamma_{\gamma}\right)$ whose composition $h$ conjugates $G_{2}$ to a symmetry group $\widehat{G}_{2}$ of $\Gamma_{\gamma}$ equivalent to $G_{1}$ relative to $N\left(\Gamma_{\gamma}\right)$. By a $G_{2}$-equivariant isotopy, we may assume that these incompressible tori are disjoint from $\mathscr{B}_{2}$. Then $h$ restricts to the identity map on $\mathscr{B}_{2}$. Suppose that $H \in \operatorname{Diff}\left(S^{3}, \Gamma_{\gamma}\right)$ realizes the above equivalence of $\widehat{G}_{2}$ and $G_{1}$. Then $H$ takes $\operatorname{Sing}\left(\widehat{G}_{2}\right)$ to $\operatorname{Sing}\left(G_{1}\right)$. As a consequence of the affirmative 
answer to the Smith conjecture [15], $\operatorname{Sing}\left(G_{1}\right)$ is either an empty set, a trivial knot, or a Hopf link whose components have different indices. Suppose that the orientation of $\operatorname{Sing}\left(G_{1}\right)$ is induced from the orientation of $\operatorname{Sing}\left(\widehat{G}_{2}\right)$ by $H$.

Suppose that $B_{2, j}$ and $B_{2, k}$ are connected by an $\operatorname{arc} \beta \operatorname{in} \operatorname{Sing}\left(\widehat{G}_{2}\right)-\operatorname{int} \mathscr{B}_{2}$, and that $\operatorname{Sing}\left(\widehat{G}_{2}\right)$ meets $B_{2, k}$ in an arc $\delta$. Then $B_{2, k}$ can be modified by a $\widehat{G}_{2}$-equivariant deformation along $\beta \cup B_{2, j}$ similar to the inverse of that mentioned above so as to contain $B_{2, j}$. Moreover, it can be deformed along $\beta \cup \delta \cup B_{2, j}$ so as to avoid $B_{2, j}$ again, as illustrated in Figure 5. Note that this operation changes the order in which the circle in $\operatorname{Sing}\left(\widehat{G}_{2}\right)$ containing $\beta$ meets the balls in $\left\{B_{2,1}, \ldots, B_{2, n}\right\}$.

Figure 5. Modification of $B_{2, k}$ realizing the transposition of $B_{2, j}$ and $B_{2, k}$.
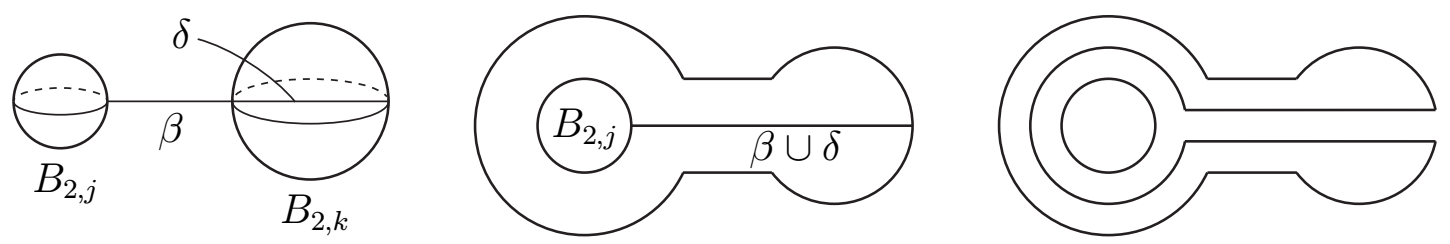

Let $C$ be a component of $\operatorname{Sing}\left(G_{1}\right)$. Without loss of generality, $C$ meets $B_{1,1}, \ldots, B_{1, r}$ in order, and avoids $B_{1, r+1}, \ldots, B_{1, n}$. Since $G_{1}$ and $G_{2}$ agree on $N(\Gamma)$, the component $H^{-1}(C)$ of $\operatorname{Sing}\left(\widehat{G}_{2}\right)$ meets $B_{2,1}, \ldots, B_{2, r}$ possibly not in order. Since every permutation on the set $\left\{B_{2,1}, \ldots, B_{2, r}\right\}$ is a product of transpositions realized by the above operation, we may assume that $H^{-1}(C)$ meets $B_{2,1}, \ldots, B_{2, r}$ in order. Apply this argument to each component of $\operatorname{Sing}\left(G_{1}\right)$. Since each $\partial \mathscr{B}_{i}$ realizes the prime factorization of $E(\Gamma)$, we can modify $H$ by a $G_{1}$-equivariant isotopy relative to $N\left(\Gamma_{\gamma}\right)$ so that we have $H\left(\operatorname{Sing}\left(\widehat{G}_{2}\right)\right)=\operatorname{Sing}\left(G_{1}\right)$ and $H\left(B_{2, j}\right)=B_{1, j}$ for each $j$. Thus, $H$ is modified so as to conjugate the action of $\widehat{G}_{2}$ on $S^{3}-\operatorname{int} \mathscr{B}_{2}$ to the action of $G_{1}$ on $S^{3}-\operatorname{int} \mathscr{B}_{1}$.

After this modification, $H$ restricts to an orientation-preserving homeomorphism on $\mathscr{B}_{1}$. Therefore, $H\left(\Gamma \cap \mathscr{B}_{2}\right)$ is ambient isotopic to $\Gamma \cap \mathscr{B}_{1}$ in $\mathscr{B}_{1}$. Hence, $H$ can be modified in $\mathscr{B}_{1}$ so as to restrict to the identity map on $N(\Gamma)$. This completes the proof.

Lemma 3.2. Suppose that $G_{1}$ and $G_{2}$ are orientation-preserving finite cyclic group actions on $S^{2} \times I$ such that

(1) $G_{1}$ and $G_{2}$ do not interchange the components of $S^{2} \times \partial I$, and

(2) $G_{1}$ and $G_{2}$ agree on $S^{2} \times \partial I$.

Then a rational twist along $S^{2} \times\{1\}$ conjugates $G_{2}$ to a finite group action equivalent to $G_{1}$ relative to $S^{2} \times \partial I$.

Proof. It is enough to consider the case where $G_{1}$ is not trivial. It follows from the remark after Theorem 8.1 of [16] that $S^{2} \times I$ admits a $G_{1}$-invariant product structure $\mathscr{P}_{1}$ and a $G_{2}$-invariant product structure $\mathscr{P}_{2}$. Since the actions of $G_{1}$ and $G_{2}$ on $S^{2} \times\{0\}$ are conjugate to a rotation of $S^{2}$ (see [15]), each Fix $\left(G_{i}\right)$ consists of two $I$-fibers in $\mathscr{P}_{i}$. Since $G_{1}$ and $G_{2}$ agree on $S^{2} \times \partial I$, we have $\partial \operatorname{Fix}\left(G_{1}\right)=\partial \operatorname{Fix}\left(G_{2}\right)$.

Denote by $p_{i}: S^{2} \times I \rightarrow S^{2} \times I / G_{i}$ the projection map onto the quotient space for each $i$, and by $S_{t}$ the $S^{2}$-fiber $S^{2} \times\{t\}$ in $\mathscr{P}_{1}$. Connect the two cone points of $p_{1}\left(S_{0}\right)$ by an arc $\bar{a}$ embedded in $p_{1}\left(S_{0}\right)$. 
Then $p_{1}^{-1}(\bar{a})$ is a spatial $\theta_{n}$-curve consisting of two vertices on the fixed points and $n>1$ edges each connecting them. Denote by $A_{i}$ the branched surface consisting of $I$-fibers in $\mathscr{P}_{i}$ attaching $p_{1}^{-1}(\bar{a})$ for each $i$. Then each $p_{1}\left(A_{i} \cap S_{1}\right)$ is an arc connecting the two cone points on $p_{1}\left(S_{1}\right)$. Since the underlying space of $p_{1}\left(S_{1}\right)$ is a sphere, $p_{1}\left(A_{2} \cap S_{1}\right)$ is isotopic to $p_{1}\left(A_{1} \cap S_{1}\right)$ relative to the cone points. Therefore, $A_{2}$ is deformed by a $G_{2}$-equivariant isotopy relative to $S_{0}$ so that $A_{1} \cap S_{1}=A_{2} \cap S_{1}$. There are two cases depending on whether $\operatorname{Fix}\left(G_{1}\right)$ and $\operatorname{Fix}\left(G_{2}\right)$ are isotopic relative to the endpoints or not.

Assume that $\operatorname{Fix}\left(G_{1}\right)$ and $\operatorname{Fix}\left(G_{2}\right)$ are isotopic relative to the endpoints. Then $\mathcal{P}_{2}$ is deformed by an isotopy relative to $S^{2} \times \partial I$ so as to agree with $\mathcal{P}_{1}$ on a setwise $G_{1}$-invariant tubular neighborhood $N\left(\operatorname{Fix}\left(G_{1}\right)\right)$ saturated in the $I$-bundle structure induced from $\mathcal{P}_{1}$. Since each $A_{i}$ meets the solid torus $\left(S^{2} \times I\right)-\operatorname{int} N\left(\operatorname{Fix}\left(G_{1}\right)\right)$ in the system of meridian disks, $A_{2}$ is moved to $A_{1}$ by an isotopy relative to $\left(S^{2} \times \partial I\right) \cup N\left(\operatorname{Fix}\left(G_{1}\right)\right)$. We may therefore assume $A_{1}=A_{2}$, and that $G_{1}$ and $G_{2}$ agree on $N\left(\operatorname{Fix}\left(G_{1}\right)\right)$. Then the $I$-bundle structures in $\mathscr{P}_{1}$ and $\mathscr{P}_{2}$ respectively induce the orbifold isomorphisms $\varphi_{1}: p_{2}\left(S_{1}\right) \rightarrow$ $p_{2}\left(S_{0}\right)$ and $\varphi_{2}: p_{2}\left(S_{0}\right) \rightarrow p_{1}\left(S_{1}\right)$ such that $\varphi_{2} \circ \varphi_{1}$ is isotopic to the identity map by an isotopy relative to the cone points which setwise preserves $p_{2}\left(A_{2} \cap S_{1}\right)$. Then we can deform $\mathscr{P}_{2}$ by an isotopy on $p_{2}\left(S^{2} \times I\right)$ relative to $p_{2}\left(S_{0}\right)$ which setwise preserves $p_{2}\left(A_{2} \cap S_{1}\right)$ so that $\mathscr{P}_{1}$ and $\mathscr{P}_{2}$ induce the same $\partial I$-bundle structure on $S^{1} \times \partial I$. Hence, the diffeomorphism of $S^{2} \times I$ which takes $\mathscr{P}_{2}$ to $\mathscr{P}_{1}$ induces the equivalence of $G_{1}$ and $G_{2}$ relative to $S^{2} \times \partial I$, as required.

Assume that $\operatorname{Fix}\left(G_{1}\right)$ and $\operatorname{Fix}\left(G_{2}\right)$ are not isotopic relative to the endpoints. Let $h: S_{1} \rightarrow S_{1}$ be a lift of an orientation-preserving involution on $p_{1}\left(S_{1}\right)$ which interchanges the cone points. Then $h$ is a diffeomorphism isotopic to the identity map which conjugates the action of $G_{2}$ on $S_{1}$ to itself and is realized by a $1 / 2$-twist along the sphere $S_{1}$. We may therefore assume that $\operatorname{Fix}\left(G_{1}\right)$ and $\operatorname{Fix}\left(G_{2}\right)$ are isotopic relative to the endpoints. Hence, the conclusion follows by the argument presented for the previous case.

Proof of Theorem 1.1. It is enough by Proposition 2.5 to prove the theorem in the case where $\Gamma$ is splittable. Then $G_{1}$ and $G_{2}$ are cyclic groups acting on $\Gamma$ freely. We may assume by Lemma 3.1 that there is a setwise $G_{1}$-invariant and setwise $G_{2}$-invariant system $\mathscr{B}$ of disjoint balls in $S^{3}$ not containing $\gamma$ such that $\partial \mathscr{B}$ realizes the prime factorization of $E(\Gamma)$, and that $G_{1}$ and $G_{2}$ agree on $E(\mathscr{B})$.

Suppose that $\mathscr{B}$ consists of balls $B_{1}, \ldots, B_{n}$. Each $\Gamma \cap B_{i}$ is a non-empty, non-splittable, spatial subgraph of $\Gamma$. By applying Proposition 2.5 to the actions of the setwise stabilisers of $B_{i}$ in $G_{1}$ and $G_{2}$ on $B_{i}$, we may assume that $G_{1}$ and $G_{2}$ agree on $E(\partial \mathscr{B})$. Hence the conclusion follows by applying Lemma 3.2 to the actions of $G_{1}$ and $G_{2}$ on $N(\partial \mathscr{B})$ equivariantly.

Remark 3.3. Theorem 1.1 requires the spatial graph $\Gamma$ to have no companion knot, and the symmetry groups $G_{1}$ and $G_{2}$ of $\Gamma$ to act on $\Gamma$ freely if $\Gamma$ is splittable. These requirements are needed because of the following examples.

(1) Suppose that $\Gamma$ is a granny knot. Then $\Gamma$ has two companion knots $K_{1}$ and $K_{2}$, both of which are trefoil knots. We obtain $E\left(K_{1}\right), E\left(K_{2}\right)$, and a 2-fold composing space by the JSJ decomposition of $E(\Gamma)$. Figure 6 illustrates $\mathbb{Z}_{2}$-symmetries $G_{1}$ and $G_{2}$ of $\Gamma$ such that $G_{2}$ interchanges $E\left(K_{1}\right)$ and $E\left(K_{2}\right)$ but $G_{1}$ does not. By conjugating $G_{1}$ by a map in $\operatorname{Diff}\left(S^{3}\right)$ which moves $N(\Gamma)$ in the longitudinal direction, $G_{1}$ and $G_{2}$ are not equivalent but agree on $\partial N(\Gamma)$. Moreover, any rational 
twists along incompressible tori in $E(\Gamma)$ cannot change the induced symmetries of $E\left(K_{1}\right)$ and $E\left(K_{2}\right)$, since the trefoil knot exterior is atoroidal.

Figure 6. $\mathbb{Z}_{2}$-symmetries of a spatial graph with companion knots.
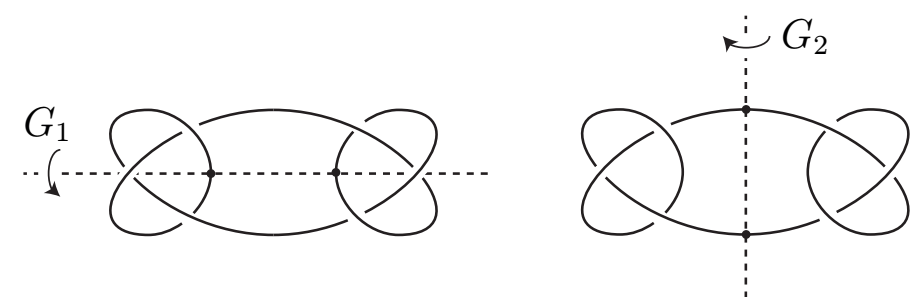

(2) Suppose that $\Gamma$ is a spatial graph which splits into non-splittable spatial graphs $\gamma_{1}, \gamma_{2}$ and $\gamma_{3}$, as illustrated in Figure 7, where $\gamma_{1}$ is a spatial $\theta$-curve. According to the choice of two edges of $\gamma_{1}$, we obtain a trefoil knot $K_{1}$, a figure-eight knot $K_{2}$, or their connected sum $K_{1} \# K_{2}$. Then any map in $\operatorname{Diff}\left(S^{3}, \Gamma\right)$ does not permute these edges. The $\mathbb{Z}_{2}$-symmetries $G_{1}$ and $G_{2}$ of $\Gamma$ illustrated in Figure 7 are not equivalent, since there is no map in $\operatorname{Diff}\left(S^{3}, \Gamma\right)$ which takes $\operatorname{Sing}\left(G_{1}\right)$ to $\operatorname{Sing}\left(G_{2}\right)$ and interchanges $\gamma_{2}$ and $\gamma_{3}$. Moreover, we cannot perform rational twists along incompressible spheres and tori in $E(\Gamma)$ to make $G_{2}$ equivalent to $G_{1}$, since any setwise $G_{2}$-invariant incompressible sphere in $E(\Gamma)$ separates $\gamma_{2}$ and $\gamma_{3}$.

Figure 7. $\mathbb{Z}_{2}$-symmetries which are not free on a splittable spatial graph.
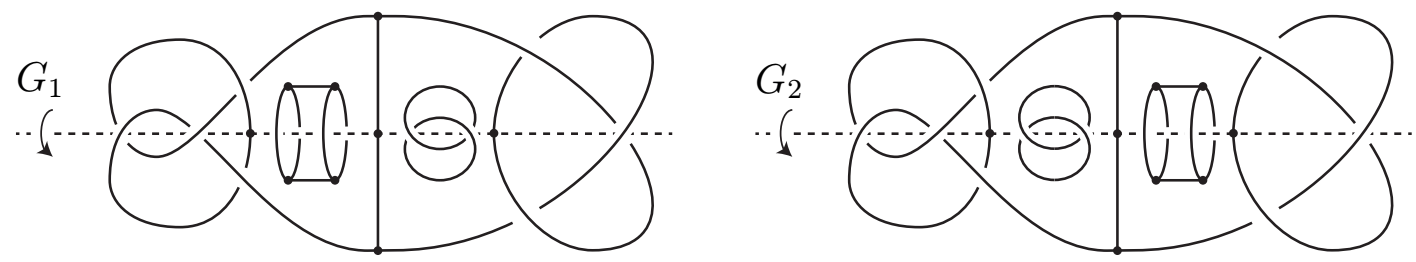

\section{Acknowledgements}

The author would like to thank the referees for helpful comments which improved this paper.

\section{References}

1. Simon, J. Topological chirality of certain molecules. Topology 1986, 25, 229-235.

2. Flapan, E. Symmetries of Möbius ladders. Math. Ann. 1989, 283, 271-283.

3. Flapan, E. Rigidity of graph symmetries in the 3-sphere. J. Knot Theor. Ramif. 1995, 4, 373-388.

4. Flapan, E.; Naimi, R.; Pommersheim, J.; Tamvakis, H. Topological symmetry groups of graphs embedded in the 3-sphere. Comment. Math. Helv. 2005, 80, 317-354.

5. Flapan, E.; Naimi, R.; Tamvakis, H. Topological symmetry groups of complete graphs in the 3-sphere. J. London Math. Soc., 2006, 73, 237-251.

6. Noda, C. The topological symmetry group of a canonically embedded complete graph in $S^{3}$. Tokyo J. Math. 1997, 20, 45-50. 
7. Flapan, E. Infinitely periodic knots. Cana. J. Math. 1985, 37, 17-28.

8. Flapan, E. The finiteness theorem for symmetries of knots and 3-manifolds with nontrivial characteristic decompositions. Topol. Appl. 1986, 24, 123-131.

9. Boileau, M.; Flapan, E. Uniqueness of free actions on $S^{3}$ respecting a knot. Can. J. Math. 1987, 39, 969-982.

10. Sakuma, M. Uniqueness of symmetries of knots. Math. Z. 1986, 192, 225-242.

11. Ikeda, T. Finite group actions on homologically peripheral 3-manifolds. Math. Proc. Cambridge Philos. Soc. 2011, 151, 319-337.

12. Jaco, W. Lectures on three manifold topology; CBMS Regional Conference Series in Mathematic 43; American Mathematical Society: Providence, RI, USA, 1980.

13. Jaco, W.; Shalen, P. Seifert fibered spaces in 3-manifolds; Memoirs of the American Mathematical Society 220: Providence, RI, USA, 1979.

14. Johannson, K. Homotopy equivalences of 3-manifolds with boundaries; Lecture Notes in Mathmatics 761; Springer: Berlin, Germany, 1979.

15. Morgan, J.W.; Bass, H. Eds. The Smith conjecture; Pure and Applied Mathematics 112; Academic Press Inc.: Orlando, FL, USA, 1984.

16. Meeks, W.H.; Scott, P. Finite group actions on 3-manifolds. Invent. Math. 1986, 86, 287-346.

17. Scott, P. The geometries of 3-manifolds. Bull. Lond. Math. Soc. 1984, 15,401-487.

18. Dinkelbach, J.; Leeb, B. Equivariant Ricci flow with surgery and applications to finite group actions on geometric 3-manifolds. Geom. Topol. 2009, 13, 1129-1173.

19. Benedetti, R.; Petronio, C. Lectures on hyperbolic geometry; Universitext; Springer-Verlag: Berlin, Germany, 1992.

20. Newman, W.H.A. A theorem on periodic transformations of spaces. Quart. J. Math. 1931, 2, 1-8.

21. Meeks, W.H.; Yau, S.T. The equivariant Dehn's lemma and loop theorem. Comment. Math. Helvetici 1981, 56, 225-239.

22. Edmonds, A.L. A topological proof of the equivariant Dehn lemma. Trans. Am. Math. Soc. 1986, 297, 605-615.

23. Plotnick, S.P. Finite group actions and nonseparating 2-spheres. Proc. Am. Math. Soc. 1984, 90, 430-432.

(c) 2012 by the author; licensee MDPI, Basel, Switzerland. This article is an open access article distributed under the terms and conditions of the Creative Commons Attribution license (http://creativecommons.org/licenses/by/3.0/.) 